International Mathematical Forum, 2, 2007, no. 56, 2759 - 2770

\title{
Application of Variational Iteration Method to a General Riccati Equation
}

\author{
B. Batiha, M. S. M. Noorani and I. Hashim \\ School of Mathematical Sciences \\ National University of Malaysia \\ 43600 UKM Bangi Selangor, Malaysia \\ belalbatiha2002@yahoo.com \\ msn@ukm.my \\ ishak_h@ukm.my
}

\begin{abstract}
In this paper, the variational iteration method (VIM) is applied to the solution of general Riccati differential equations. The equations under consideration includes one with variable coefficient and one in matrix form. In VIM, a correction functional is constructed by a general Lagrange multiplier which can be identified via a variational theory. The VIM yields an approximate solution in the form of a quickly convergent series. Comparisons with exact solution and the fourth-order RungeKutta method show that the VIM is a powerful method for the solution of nonlinear equations. The present paper may be a suitable and fruitful exercise for teaching and better understanding techniques in advanced undergraduate courses on classical mechanics.
\end{abstract}

Mathematics Subject Classification: 65L05, 65L06, 35A15

Keywords: Riccati equation, Variational iteration method, Runge-Kutta method

\section{Introduction}

The Riccati differential equation is named after the Italian nobleman Count Jacopo Francesco Riccati (1676-1754). The book of Reid [22] contains the fundamental theories of Riccati equation, with applications to random processes, 
optimal control, and diffusion problems. Besides important engineering science applications that today are considered classical, such as stochastic realization theory, optimal control, robust stabilization, and network synthesis, the newer applications include such areas as financial mathematics [3, 18]. The solution of this equation can be reached using classical numerical methods such as the forward Euler method and Runge-Kutta method. An unconditionally stable scheme was presented by Dubois and Saidi [7]. El-Tawil et al. [4] presented the usage of Adomian decomposition method (ADM) to solve the nonlinear Riccati in an analytic form. Very recently, Tan and Abbasbandy [24] employed the analytic technique called Homotopy Analysis Method (HAM) to solve a quadratic Riccati equation.

The variational iteration method (VIM) is a simple and yet powerful method for solving a wide class of nonlinear problems, first envisioned by He [11] (see also $[13,15,16,12,14])$. The VIM has successfully been applied to many situations. For example, He [13] solved the classical Blasius' equation using VIM. He [15] used VIM to give approximate solutions for some well-known non-linear problems. He [16] used VIM to solve autonomous ordinary differential systems. He [12] coupled the iteration method with the perturbation method to solve the well-known Blasius equation. He [14] solved strongly nonlinear equations using VIM. Soliman [23] applied the VIM to solve the KdV-Burger's and Lax's seventh-order KdV equations. The VIM has recently been applied for solving nonlinear coagulation problem with mass loss by Abulwafa et al. [2]. Momani et al. The VIM has been applied for solving nonlinear differential equations of fractional order by Odibat et al. [20]. Bildik et al. [5] used VIM for solving different types of nonlinear partial differential equations. Dehghan and Tatari [6] employed VIM to solve a Fokker-Planck equation. Wazwaz [25] presented a comparative study between the variational iteration method and Adomian decomposition method. Tamer et al. [8] introduced a modification of VIM. Batiha et al. [?] used VIM to solve the generalized Burgers-Huxley equation. Batiha et al. [?] applied VIM to the generalized Huxley equation. Junfeng [17] introduced Variational iteration method to solve two-point boundary value problems. Abbasbandy [1] solved one example of the quadratic Riccati differential equation (with constant coefficient) by He's variational iteration method by using Adomian's polynomials.

The aim of this paper, is to continue the analysis of VIM on different types of Riccati differential equations. In particular, we give 5 examples of Riccati differential equations which include one with variable coefficient and one in matrix form. Numerical comparison between VIM, RK4 and exact solution on these equations are given. We think that, this paper can be used to convey to students the idea that the VIM is a powerful tools for approximately solving linear and nonlinear 


\section{Variational iteration method}

This method, which is a modified general Lagrange's multiplier method [10], has been shown to solve effectively, easily and accurately a large class of nonlinear problems $[11,13,15,16,12,14,23,2,20,5]$. The main feature of the method is that the solution of a mathematical problem with linearization assumption is used as initial approximation or trial function. Then a more highly precise approximation at some special point can be obtained. This approximation converges rapidly to an accurate solution. To illustrate the basic concepts of the VIM, we consider the following nonlinear differential equation:

$$
L u+N u=g(x),
$$

where $L$ is a linear operator, $N$ is a nonlinear operator, and $g(x)$ is an inhomogeneous term. According to the VIM [15, 16, 12, 14], we can construct a correction functional as follows:

$$
u_{n+1}(x)=u_{n}(x)+\int_{0}^{x} \lambda\left\{L u_{n}(\tau)+N \tilde{u}_{n}(\tau)-g(\tau)\right\} \mathrm{d} \tau,
$$

where $\lambda$ is a general Lagrangian multiplier [10], which can be identified optimally via the variational theory, the subscript $n$ denotes the $n$ th-order approximation, $\tilde{u}_{n}$ is considered as a restricted variation $[15,16,12]$, i.e. $\delta \tilde{u}_{n}=0$.

\section{Analysis of general Riccati differential equa- tion}

In this paper, we present numerical and analytical solutions for the general Riccati differential equation [21]:

$$
\frac{d y}{d t}=Q(t) y+R(t) y^{2}+P(t), \quad y(0)=G(t),
$$

where $Q(t), R(t), P(t)$ and $G(t)$ are scalar functions. To solve equation (3) by means of He's variational iteration method, we construct a correction functional,

$$
\begin{aligned}
y_{n+1}(t)= & y_{n}(t)+\int_{0}^{t} \lambda(s)\left[\frac{d y_{n}(s)}{\mathrm{d} s}-Q(s) \tilde{y}_{n}(s)\right. \\
& \left.-R(s) \tilde{y}_{n}^{2}(s)-P(s)\right] \mathrm{d} s \\
\delta y_{n+1}(t)= & \delta y_{n}(t)+\delta \int_{0}^{t} \lambda(s)\left[\frac{d y_{n}(s)}{\mathrm{d} s}-Q(s) \tilde{y}_{n}(s)\right.
\end{aligned}
$$




$$
\begin{gathered}
\left.-R(s) \tilde{y}_{n}^{2}(s)-P(s)\right] \mathrm{d} s \\
\delta y_{n+1}(t)=\delta y_{n}(t)+\delta \int_{0}^{t} \lambda(s)\left[\frac{d y_{n}(s)}{\mathrm{d} s}\right] \mathrm{d} s, \\
\delta y_{n+1}(t)=(1+\lambda) \delta y_{n}(t)-\int_{0}^{t} \delta y_{n}(s) \lambda^{\prime} \mathrm{d} s=0,
\end{gathered}
$$

where $\tilde{y}_{n}$ is considered as restricted variations, which mean $\delta \tilde{y}_{n}=0$. Its stationary conditions can be obtained as follows

$$
1+\lambda(t)=0,\left.\quad \lambda^{\prime}(s)\right|_{s=t}=0 .
$$

The Lagrange multipliers, therefore, can be identified as $\lambda(s)=-1$ and the following variational iteration formula is obtained

$$
\begin{aligned}
y_{n+1}(t)= & y_{n}(t)-\int_{0}^{t}\left[\frac{d y_{n}(s)}{\mathrm{d} s}-Q(s) y_{n}(s)\right. \\
& \left.-R(s) y_{n}^{2}(s)-P(s)\right] \mathrm{d} s .
\end{aligned}
$$

\section{Numerical examples}

\subsection{Example 1}

Consider the following example:

$$
\frac{\mathrm{d} y}{\mathrm{~d} t}=-y^{2}(t)+1, \quad y(0)=0 .
$$

Here $Q(t)=0, R(t)=-1, P(t)=1$ and $G(t)=0$. The exact solution was found to be [4]:

$$
y(t)=\frac{\mathrm{e}^{2 t}-1}{\mathrm{e}^{2 t}+1} .
$$

To solve equation (6) by means of He's variational iteration method, we construct a correction functional (see (5)),

$$
y_{n+1}(t)=y_{n}(t)-\int_{0}^{t}\left[\frac{\mathrm{d} y_{n}}{\mathrm{~d} s}+y_{n}^{2}-1\right] \mathrm{d} s .
$$

We can take the linearized solution $y(t)=t+C$ as the initial approximation $y_{0}$, the condition $y(0)=0$ gives us $C=0$. Then we get:

$$
y_{1}(t)=t-\frac{1}{3} t^{3}
$$




$$
\begin{aligned}
y_{2}(t)= & t-\frac{1}{3} t^{3}+\frac{2}{15} t^{5}-\frac{1}{63} t^{7} \\
y_{3}(t)= & t-\frac{1}{3} t^{3}+\frac{2}{15} t^{5}-\frac{1}{63} t^{7}+\frac{38}{2835} t^{9} \\
& -\frac{134}{51975} t^{11}+\frac{4}{12285} t^{13}-\frac{1}{59535} t^{15}
\end{aligned}
$$

In the same manner, the rest of the components of the iteration formulae (8) can be obtained using the Maple Package.

\subsection{Example 2}

Consider this simple example:

$$
\frac{\mathrm{d} y}{\mathrm{~d} t}=2 y(t)-y^{2}(t)+1, \quad y(0)=0 .
$$

Here $Q(t)=2, R(t)=-1, P(t)=1$ and $G(t)=0$.

The exact solution was found to be [4]:

$$
y(t)=1+\sqrt{2} \tanh \left(\sqrt{2} t+\frac{1}{2} \log \left(\frac{\sqrt{2}-1}{\sqrt{2}+1}\right)\right) .
$$

To solve equation (9) using VIM, we construct a correction functional (see (5)),

$$
y_{n+1}(t)=y_{n}(t)-\int_{0}^{t}\left[\frac{\mathrm{d} y_{n}}{\mathrm{~d} s}-2 y_{n}+y_{n}^{2}-1\right] \mathrm{d} s .
$$

We can take the linearized solution $y(t)=-\frac{1}{2}+\mathrm{e}^{2 t} C$ as the initial approximation $y_{0}$, the condition $y_{0}$ gives us $C=0.5$. Then we get:

$$
\begin{aligned}
y_{1}(t)= & -0.6875+0.75 \mathrm{e}^{2 t}-0.0625 \mathrm{e}^{4 t}-\frac{1}{8} \ln \mathrm{e}^{2 t} \\
y_{2}(t)= & -0.995605468750+0.75 \mathrm{e}^{2 t}-0.0625 \mathrm{e}^{4 t}-\frac{1}{8} \ln \mathrm{e}^{2 t} \\
& -0.59765625 t-0.12890625 \mathrm{e}^{4 t}-0.10546875 \ln \left(\mathrm{e}^{2 t}\right)^{2} \\
& +0.421875 \mathrm{e}^{2 t}+0.015625 \mathrm{e}^{6 t}+0.09375 \mathrm{e}^{2 t} \ln \mathrm{e}^{2 t} \\
& -0.00260416666667 \ln \left(\mathrm{e}^{2 t}\right)^{3}-0.00390625 \mathrm{e}^{4 t} \ln \mathrm{e}^{2 t} \\
& -0.00048828125 \mathrm{e}^{8 t} .
\end{aligned}
$$

Again, the rest of the components of the iteration formulae (11) can be obtained using the Maple Package. 


\subsection{Example 3}

Consider the example [19]:

$$
\frac{\mathrm{d} y}{\mathrm{~d} t}=t^{2}+y^{2}(t), \quad y(0)=1
$$

where $Q(t)=0, R(t)=1, P(t)=t^{2}$ and $G(t)=1$. To solve equation (12) using variational iteration method, we construct a correction functional,

$$
y_{n+1}(t)=y_{n}(t)-\int_{0}^{t}\left[\frac{\mathrm{d} y_{n}}{\mathrm{~d} s}-s^{2}+y_{n}^{2}\right] \mathrm{d} s .
$$

We can take the linearized solution $y(t)=\frac{t^{3}}{3}+C$ as the initial approximation $y_{0}$, the condition $y(0)=1$ gives us $C=1$. Then we get:

$$
\begin{aligned}
y_{1}(t)= & \frac{1}{63} t^{7}+\frac{1}{6} t^{4}+t^{3}+t+1 \\
y_{2}(t)= & t+t^{2}+\frac{4}{3} t^{3}+\frac{1}{2} t^{4}+\frac{7}{15} t^{5}+\frac{1}{18} t^{6}+\frac{1}{7} t^{7}+\frac{23}{504} t^{8} \\
& +\frac{5}{756} t^{9}+\frac{2}{693} t^{11}+\frac{1}{2268} t^{12}+\frac{1}{59535} t^{15}+1 .
\end{aligned}
$$

The rest of the components of the iteration formulae (13) can be obtained using the Maple Package.

\subsection{Example 4}

Consider the following variable coefficient example:

$$
\frac{\mathrm{d} y}{\mathrm{~d} t}=t^{3} y^{2}(t)-2 t^{4} y(t)+t^{5}+1, \quad y(0)=0
$$

where $Q(t)=-2 t^{4}, R(t)=t^{3}, P(t)=t^{5}+1$ and $G(t)=0$. The exact solution is:

$$
y(t)=t
$$

In order to solve equation (14) by using He's variational iteration method, we construct a correction functional (see (5)),

$$
y_{n+1}(t)=y_{n}(t)-\int_{0}^{t}\left[\frac{\mathrm{d} y_{n}}{\mathrm{~d} s}-s^{3} y_{n}^{2}+2 s^{4} y_{n}-s^{5}-1\right] \mathrm{d} s .
$$


As before we can take the linearized solution $y(t)=t+C$ as the initial approximation $y_{0}$, the condition $y(0)=0$ gives us $C=0$. Then we get:

$$
y_{1}(t)=t
$$

which is the exact solution.

\subsection{Example 5}

The following example of a matrix Riccati differential equation is due to [4]:

$$
\frac{\mathrm{d} y}{\mathrm{~d} t}=-y^{2}(t)+Q, \quad y(0)=0
$$

where

$$
Q=\frac{1}{2}\left(\begin{array}{cc}
1 & -1 \\
1 & 1
\end{array}\right)\left(\begin{array}{cc}
1 & 0 \\
0 & 100
\end{array}\right)\left(\begin{array}{cc}
1 & 1 \\
-1 & 1
\end{array}\right) .
$$

To find the solution of this equation via VIM, we shall treat the matrix equation as a system of differential equations. So, we rewrite Eq.(17) as system of equations:

$$
\begin{aligned}
\frac{\mathrm{d} y_{11}}{\mathrm{~d} t} & =-\left[y_{11}^{2}(t)+y_{12}(t) y_{21}(t)\right]+\frac{101}{2}, \\
\frac{\mathrm{d} y_{12}}{\mathrm{~d} t} & =-\left[y_{11}(t) y_{12}(t)+y_{12}(t) y_{22}(t)\right]-\frac{99}{2}, \\
\frac{\mathrm{d} y_{21}}{\mathrm{~d} t} & =-\left[y_{11}(t) y_{12}(t)+y_{12}(t) y_{22}(t)\right]-\frac{99}{2}, \\
\frac{\mathrm{d} y_{22}}{\mathrm{~d} t} & =-\left[y_{11}^{2}(t)+y_{12}(t) y_{21}(t)\right]+\frac{101}{2} .
\end{aligned}
$$

To solve equations (19), (20), (21) and (22) by means of He's variational iteration method, we construct a correction functional,

$$
\begin{aligned}
y_{11, n+1}(t)= & y_{11, n}(t)+\int_{0}^{t} \lambda_{11}(s)\left[\frac{\mathrm{d} y_{11, n}}{\mathrm{~d} s}+\tilde{y}_{11, n}^{2}\right. \\
& \left.+\tilde{y}_{12, n} \tilde{y}_{21, n}-\frac{101}{2}\right] \mathrm{d} s \\
y_{12, n+1}(t)= & y_{12, n}(t)+\int_{0}^{t} \lambda_{12}(s)\left[\frac{\mathrm{d} y_{12, n}}{\mathrm{~d} s}+\tilde{y}_{11, n} \tilde{y}_{12, n}\right. \\
& \left.+\tilde{y}_{12, n} \tilde{y}_{22, n}+\frac{99}{2}\right] \mathrm{d} s,
\end{aligned}
$$




$$
\begin{aligned}
y_{21, n+1}(t)= & y_{21, n}(t)+\int_{0}^{t} \lambda_{21}(s)\left[\frac{\mathrm{d} y_{21, n}}{\mathrm{~d} s}+\tilde{y}_{11, n} \tilde{y}_{12, n}\right. \\
& \left.+\tilde{y}_{12, n} \tilde{y}_{22, n}+\frac{99}{2}\right] \mathrm{d} s \\
y_{22, n+1}(t)= & y_{22, n}(t)+\int_{0}^{t} \lambda_{22}(s)\left[\frac{\mathrm{d} y_{22, n}}{\mathrm{~d} s}+\tilde{y}_{11, n}^{2}\right. \\
& \left.+\tilde{y}_{12, n} \tilde{y}_{21, n}-\frac{101}{2}\right] \mathrm{d} s,
\end{aligned}
$$

where $\tilde{y}_{i j, n}$ are considered as restricted variation i.e. $\delta y_{i j, n}=0$. Its stationary conditions can be obtained as:

$$
\begin{array}{ll}
1+\lambda_{11}(t)=0, & \left.\lambda_{11}^{\prime}(s)\right|_{s=t}=0 \\
1+\lambda_{12}(t)=0, & \left.\lambda_{12}^{\prime}(s)\right|_{s=t}=0 \\
1+\lambda_{21}(t)=0, & \left.\lambda_{21}^{\prime}(s)\right|_{s=t}=0 \\
1+\lambda_{22}(t)=0, & \left.\lambda_{22}^{\prime}(s)\right|_{s=t}=0 .
\end{array}
$$

Thus, the Lagrange multipliers are $\lambda_{11}(s), \lambda_{12}(s), \lambda_{21}(s)$ and $\lambda_{22}(s)=-1$.

We can take the linearized solution $y_{11}(t)=\frac{101 t}{2}+A, y_{12}(t)=\frac{-99 t}{2}+B$, $y_{21}(t)=\frac{-99 t}{2}+C$ and $y_{11}(t)=\frac{101 t}{2}+D$ as the initial approximation, the condition $y(0)=0$ gives us $A, B, C$ and $D=0$. Then we get:

$$
\begin{aligned}
& y_{11,1}(t)=101 t-\frac{10001}{2} t^{3}, \\
& y_{12,1}(t)=-99 t+\frac{9999}{2} t^{3}, \\
& y_{21,1}(t)=-99 t+\frac{9999}{2} t^{3}, \\
& y_{22,1}(t)=101 t-\frac{10001}{2} t^{3} .
\end{aligned}
$$

In the same manner, the rest of the components of the iteration formulae can be obtained using the Maple Package.

\section{Numerical results and discussion}

We now obtain numerical solutions of Riccati differential equation. Table 1 shows comparison between the 3-iterate of VIM and the exact solution for example 1. Table 2 shows comparison between the 3 -iterate of VIM and the exact solution for example 2. Table 3 shows comparison between the 2-iterate of VIM, Taylor matrix, Runge-Kutta, Picard and Euler method. Table 4 shows comparison between the 8-iterate VIM and RK4 for example 5. In Table 4 we use the Padé approximation [10,10] for 8-iterate VIM. 
Table 1: Numerical comparisons for example 1

\begin{tabular}{cccc}
\hline$t$ & Exact solution & 3-Iterate VIM & absolute error \\
\hline 0.1 & 0.0996679946 & 0.09966799461 & $5.000 \mathrm{E}-11$ \\
0.2 & 0.1973753203 & 0.1973753160 & $4.300 \mathrm{E}-9$ \\
0.3 & 0.2913126124 & 0.2913124564 & $1.560 \mathrm{E}-7$ \\
0.4 & 0.3799489622 & 0.3799469862 & $1.976 \mathrm{E}-6$ \\
0.5 & 0.4621171572 & 0.4621033328 & $1.382 \mathrm{E}-5$ \\
0.6 & 0.5370495670 & 0.5369833784 & $6.619 \mathrm{E}-5$ \\
0.7 & 0.6043677771 & 0.6041244734 & $2.433 \mathrm{E}-4$ \\
0.8 & 0.6640367702 & 0.6633009217 & $7.358 \mathrm{E}-4$ \\
0.9 & 0.7162978702 & 0.7143823394 & $1.916 \mathrm{E}-3$ \\
1.0 & 0.7615941560 & 0.7571662670 & $4.428 \mathrm{E}-3$ \\
\hline
\end{tabular}

Table 2: Numerical comparisons for example 2

\begin{tabular}{cccc}
\hline$t$ & Exact solution & 3-Iterate VIM & absolute error \\
\hline 0.1 & 0.1102951967 & 0.1102952165 & $1.980 \mathrm{E}-8$ \\
0.2 & 0.2419767992 & 0.2419778311 & $1.032 \mathrm{E}-6$ \\
0.3 & 0.3951048481 & 0.3951137053 & $8.857 \mathrm{E}-6$ \\
0.4 & 0.5678121656 & 0.5678455104 & $3.334 \mathrm{E}-5$ \\
0.5 & 0.7560143925 & 0.7560869922 & $7.260 \mathrm{E}-5$ \\
0.6 & 0.9535662155 & 0.9536660314 & $9.982 \mathrm{E}-5$ \\
0.7 & 1.1529489660 & 1.1530374490 & $8.848 \mathrm{E}-5$ \\
0.8 & 1.3463636550 & 1.3463791070 & $1.545 \mathrm{E}-5$ \\
0.9 & 1.5269113120 & 1.5264118730 & $4.994 \mathrm{E}-4$ \\
1.0 & 1.6894983900 & 1.6860271340 & $3.471 \mathrm{E}-3$ \\
\hline
\end{tabular}

Table 3: Numerical comparisons for example 3

\begin{tabular}{cccc}
\hline Method & $t=0.5$ & $t=0.90$ & $t=0.95$ \\
\hline Taylor matrix [19] & 1.989580000 & 4.44700000 & 4.945000000 \\
Picard [9] & 1.969000000 & 4.23100000 & 4.671000000 \\
7.183000000 & & & \\
2-iterate VIM & 1.964676823 & 4.406704915 & 4.940949522 \\
\hline
\end{tabular}


Table 4: Numerical comparisons for example 4 between 4-iterate VIM and RK4 with $h=0.001$.

\begin{tabular}{|c|c|c|c|c|c|c|c|c|}
\hline \multirow[b]{2}{*}{$t$} & \multicolumn{2}{|c|}{$y_{11}$} & \multicolumn{2}{|c|}{$y_{12}$} & \multicolumn{2}{|c|}{$y_{21}$} & \multicolumn{2}{|c|}{$y_{22}$} \\
\hline & $\overline{\text { VIM }}$ & RK4 & $\overline{\text { VIM }}$ & RK4 & $\overline{\text { VIM }}$ & $\overline{\mathrm{RK} 4}$ & $\overline{\text { VIM }}$ & $\overline{\mathrm{RK} 4}$ \\
\hline 0.0 & 0.00000 & 0.00000 & 0.00000 & 0.00000 & 0.00000 & 0.00000 & 0.00000 & 0.00000 \\
\hline 0.1 & 3.85780 & 3.85780 & -3.7581 & -3.7581 & -3.7581 & -3.7581 & 3.85780 & 3.85780 \\
\hline 0.2 & 4.91883 & 4.91883 & -4.7215 & -4.7215 & -4.7215 & -4.7215 & 4.91883 & 4.91883 \\
\hline 0.3 & 5.12093 & 5.12093 & -4.8296 & -4.8296 & -4.8296 & -4.8296 & 5.12093 & 5.12093 \\
\hline 0.4 & 5.18662 & 5.18662 & -4.8067 & -4.8067 & -4.8067 & -4.8067 & 5.18662 & 5.18662 \\
\hline 0.5 & 5.23056 & 5.23060 & -4.7685 & -4.7685 & -4.7685 & -4.7685 & 5.23056 & 5.23060 \\
\hline 0.6 & 5.26821 & 5.26846 & -4.7312 & -4.7314 & -4.7312 & -4.7314 & 5.26821 & 5.26846 \\
\hline 0.7 & 5.30126 & 5.30218 & -4.6971 & -4.6978 & -4.6971 & -4.6978 & 5.30126 & 5.30218 \\
\hline 0.8 & 5.32945 & 5.33202 & -4.6660 & -4.6680 & -4.6660 & -4.6680 & 5.32945 & 5.33202 \\
\hline 0.9 & 5.35223 & 5.35815 & -4.6372 & -4.6419 & -4.6372 & -4.6419 & 5.35223 & 5.35815 \\
\hline 1.0 & 5.36903 & 5.38080 & -4.6100 & -4.6192 & -4.6100 & -4.6192 & 5.36903 & 5.38080 \\
\hline
\end{tabular}

\section{Conclusions}

In this paper, variation iteration method (VIM) has been successfully applied to find the approximate solution of the general Riccati differential equation. The method was used in a direct way without using linearization, perturbation or restrictive assumptions. It may be concluded that VIM is very powerful and efficient in finding analytical as well as numerical solutions for wide classes of linear and nonlinear differential equations. It provides more realistic series solutions that converge very rapidly in real physical problems. Therefore, it could be easily included in lectures on classical mechanics for undergraduate students.

\section{Acknowledgement}

The financial support received from the Academy of Sciences Malaysia under the SAGA grant no. P24c is gratefully acknowledged.

\section{References}

[1] S. Abbasbandy, A new application of He's variational iteration method for quadratic Riccati differential equation by using Adomian's polynomials. J. Comput. Appl. Math. doi:10.1016/j.cam.2006.07.012. 
[2] E.M. Abulwafa, M.A. Abdou and A.A. Mahmoud, The solution of nonlinear coagulation problem with mass loss. Chaos Solitons Fractals doi:10.1016/j.chaos.2005.08.044.

[3] B.D. Anderson and J.B. Moore, Optimal control-linear quadratic methods. Prentice-Hall, New Jersey, 1999.

[4] A.A. Bahnasawi ,M.A. El-Tawil and A. Abdel-Naby, Solving Riccati differential equation using Adomian's decomposition method. Appl. Math. Comput. (2004), 157: 503-514.

[5] N. Bildik and A.Konuralp, The use of variational iteration method, differential transform method and Adomian decomposition method for solving different types of nonlinear partial differential equations. Int. J. Nonlinear. Sci. Numer. Simul. (2006), 7: 65-70.

[6] M. Dehghan and M. Tatari, The use of He's variational iteration method for solving a Fokker-Planck equation. Phys. Scr. (2006), 74: 310-316.

[7] F. Dubois and A. Saidi, Unconditionally stable scheme for Riccati equation. ESAIM Proceedings (2000),8:39-52.

[8] H. El-Zoheiry, T.A. Abassy and M.A. El-Tawil, Toward a modified variational iteration method. J. Comput. Appl. Math. doi:10.1016/j.cam.2006.07.019.

[9] W.B. Fu, A comparison of numerical and analytical methods for the solution of a Riccati equation. Int. J. Math. Educ. Sci. Tech. (1989), 20: $421 ?-427$.

[10] M. Inokuti, H. Sekine and T. Mura, General use of the Lagrange multiplier in nonlinear mathematical physics. In: Nemat-Nassed S, editor. Variational method in the mechanics of solids. Pergamon Press, 1978.

[11] J.H. He, A new approach to nonlinear partial differential equations. Commun. Nonlin. Sci. Numer. Simul. (1997),2: 230-?235.

[12] J.H. He, A simple perturbation approach to Blasius equation. Appl. Math. Comput. (2003), 140: 217-222.

[13] J.H. He, Approximate analytical solution of Blasius' equation. Commun. Nonlin. Sci. Numer. Simul. (1998), 3: 260-263.

[14] J.H. He, Some asymptotic methods for strongly nonlinear equations. Int. J. Modern Phys. B (2006) 20: 1141-1199. 
[15] J.H. He, Variational iteration method-a kind of non-linear analytical technique: some examples. Int. J. Non-Linear Mech. (1999),34: 699-708.

[16] J.H. He, Variational iteration method for autonomous ordinary differential systems. Appl. Math. Comput. (2000), 114:115?-123.

[17] L. Junfeng, Variational iteration method for solving two-point boundary value problems. J. Comput. Appl. Math. doi:10.1016/j.cam.2006.07.014.

[18] I. Lasiecka and R.Triggiani, Differential and algebraic Riccati equations with application to boundary/point control problems: continuous theory and approximation theory (Lecture notes in control and information sciences, Vol. 164). Berlin: Springer.; 1991.

[19] G. Mustafa and S. Mehmet, On the solution of the Riccati equation by the Taylor matrix method. Appl Math Comput (2006), 176:414?-421.

[20] Z.M. Odibat and S. Momani, Application of variational iteration method to nonlinear differential equations of fractional order. Int. J. Nonlinear Sci. Numer. Simul. (2006), 7: 27-34.

[21] A.D. Polyanin and V.F. Zaitsev, Handbook of exact solutions for ordinary differential equations. Florida: CRC. 2003.

[22] W.T. Reid, Riccati differential equations (Mathematics in science and engineering, Vol. 86). New York: Academic Press. 1972.

[23] A.A. Soliman, A numerical simulation and explicit solutions of KdVBurgers' and Lax's seventh-order KdV equations. Chaos Solitons Fractals doi:10.1016/j.chaos.2005.08.054.

[24] Y. Tan and S. Abbasbandy, Homotopy analysis method for quadratic Riccati differential equation. Commun. Nonlin. Sci. Numer. Simul. doi:10.1016/j.cnsns.2006.06.006.

[25] A.M. Wazwaz, A comparison between the variational iteration method and Adomian decomposition method. J. Comput. Appl. Math. doi:10.1016/j.cam.2006.07.018.

\section{Received: March 17, 2007}

